Check for updates

Cite this: RSC Adv., 2019, 9, 11649

\title{
Preparation of biodiesel oil-in-water nanoemulsions by mixed surfactants for bifenthrin formulation
}

\author{
Huiqiong Yan, $\hat{\dagger}^{\mathrm{ab}}$ Chaoling Bao, $\hat{\dagger}^{\mathrm{b}}$ Xiuqiong Chen, ${ }^{\mathrm{ab}}$ Changjiang Yu, ${ }^{\mathrm{b}}$ Dulin Kong, ${ }^{\mathrm{c}}$ \\ Jianjun Shi ${ }^{\mathrm{a}}$ and Qiang Lin (DD *ab
}

Although several approaches have been reported on the development of nanoemulsions over the last few years, studies on the formation of biodiesel nanoemulsions for bifenthrin formulation by the low-energy phase inversion composition (PIC) method are still scarce. Herein, the preparation of oil-in-water (O/W) nanoemulsions suitable for pesticide application has been achieved in biodiesel by dissolving a bifenthrin/mixture of a non-ionic surfactant (NP-6) and an anionic surfactant (ABSCa)/water system by the PIC method. The mechanism of the formation of bifenthrin nanoemulsions by dripping the water phase into the oil-surfactant phase was exemplified via the pseudo-ternary phase diagram. The effects of the mass ratio of NP-6 and $\mathrm{ABSCa}, \mathrm{mROS}_{\mathrm{O}}$, stirring rate, the addition rate of water and the emulsification temperature on the mean droplet size of the nanoemulsion were investigated by dynamic light scattering (DLS). In addition, the interfacial tension and the contact angle of bifenthrin nanoemulsions for the spraying application were investigated. The insecticidal activity of bifenthrin nanoemulsions against cabbage maggots was further studied. Moreover, the emulsion stability of the bifenthrin nanoemulsions against Ostwald ripening behavior was evaluated, and the long-term stability of the bifenthrin formulation was studied by the HPLC method to assess the shelf life of the pesticide formulation. Experimental results showed that the optimum emulsification conditions for the mass ratio of NP- 6 and $\mathrm{ABSC} a, \mathrm{mRos}_{\mathrm{O}}$, stirring rate, the addition rate of water and the emulsification temperature were respectively 5/5,1.4, $8000 \mathrm{rpm}, 0.7 \mathrm{~mL} \mathrm{~min}^{-1}$ and $25^{\circ} \mathrm{C}$. The bifenthrin nanoemulsion with low interfacial tension and contact angle, easy adsorption on plant leaf surfaces and good shelf life has great potential for use as a pesticide formulation.

Received 23rd January 2019 Accepted 30th March 2019

DOI: 10.1039/c9ra00591a

rsc.li/rsc-advances particular, the oil-in-water nanoemulsions can avoid the use of a number of organic solvents and adjuvants, which are considered to be harmful to the environment ${ }^{5}{ }^{\text {therefore, they }}$ can be widely used in foods, beverages, cosmetics, agrochemicals and pharmaceuticals. ${ }^{6-10}$

Since nanoemulsions are thermodynamically unstable systems, energy input is required for their preparation. In this regard, two main approaches are currently used for the preparation of nanoemulsions: high energy and low energy emulsification methods. ${ }^{11,12}$ High-energy methods include extreme shear stirring, high-pressure homogenizers, ultrasound generators and microfluidizers, which apply intense mechanical energy to achieve the blending and crushing of oil and water phases. $^{2}$ Although the high-energy methods are common and straightforward approaches to fabricating nanoemulsions, the level of energy required to obtain nanometer-scaled droplets is very high and thus cost-inefficient. ${ }^{13}$ Conversely, low-energy emulsification methods, based on the spontaneous formation of nanoemulsions by taking advantage of the internal chemical energy of the system, are energy-efficient and involve phase transitions taking place during the emulsification process. ${ }^{\mathbf{1 4}}$ 
Several low-energy methods such as spontaneous phase inversion temperature (PIT) and phase inversion composition (PIC) methods have been proposed to prepare nanoemulsions, which respectively depend on specific changes in temperature at constant composition and specific changes in composition at constant temperature. ${ }^{\mathbf{1 3 , 1 5 , 1 6}}$ Although low-energy emulsification methods have been the focus of considerable research interest in the last few years, there is still a relatively poor understanding of the physicochemical basis of nanoemulsions and the major factors impacting their performance. In particular, no direct evidence has been put forward to clarify the presence of lamellar liquid crystallites or bicontinuous microemulsions in the preparation of nanoemulsions by the PIC method. Therefore, further effort is required to fully understand the mechanism of the formation of nanoemulsions, and thus optimize the emulsification process.

Biodiesel is a green environmental industrial solvent and raw material derived from renewable biomass such as vegetable oils, animal fats, waste cooking oils, lipids of yeast and microalgae. ${ }^{17}$ Due to its high biological degradation speed, high flash point, high storing and shipping safety, good environmental compatibility, low cost, relatively stronger dissolving ability and renewability, it has recently attracted much attention all over the world. ${ }^{18}$ Significantly, biodiesel can be used as the carrier for insoluble pesticides for the formation of nanoemulsions, which could improve the bioavailability of pesticide by solubilization in the droplets of nanoemulsion. ${ }^{19}$ Many works also indicate that using nanoemulsions as pesticide delivery systems could realize the efficient delivery of the active ingredients of pesticides and improve their efficacy.,20

In this study, we applied the PIC method to prepare transparent biodiesel nanoemulsions by mixed surfactants using biodiesel as the oil phase for bifenthrin formulation. It has been reported that the mixed surfactants often perform better for various applications, as compared to pure surfactant. ${ }^{21}$ For this reason, the mixture of nonionic nonylphenol polyoxyethylene ether (NP-6) and anionic calcium dodecylbenzene sulfonate (ABSCa) was used to prepare a nanoemulsion. Bifenthrin is a water-insoluble pesticide that is regarded as a thirdgeneration synthetic pyrethroid insecticide for its great photostability and insecticidal activity; ${ }^{5,22}$ its structural formula is shown in Fig. 1. The low-energy method used in our work involves constant temperature but different compositions during emulsification by adding water to an oil-surfactant mixture to obtain the oil-in-water $(\mathrm{O} / \mathrm{W})$ nanoemulsions. ${ }^{23}$ To obtain the optimum emulsification conditions we investigated the effects of the mass ratio of NP-6 and ABSCa, the mass ratio of oil and the mixed surfactant $\left(\mathrm{mR}_{\mathrm{OS}}\right)$, stirring rate, the

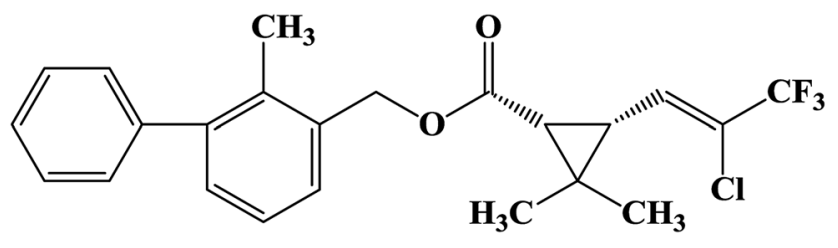

Fig. 1 Structural formula of bifenthrin. addition rate of water and the emulsification temperature on the mean droplet size of the nanoemulsion. Unlike conventional microemulsions that are thermodynamically stable systems, nanoemulsions can be kinetically stable. ${ }^{11}$ It was reported that the main breakdown process for nanoemulsions is Ostwald ripening, which is attributed to the diffusion of molecules of the disperse phase from small to big droplets in the continuous phase because of their different Laplace pressures. ${ }^{13,16}$ The emulsion stability of the nanoemulsions against Ostwald ripening behavior was evaluated, and the long-term stability of the bifenthrin formulation was also studied by the HPLC method to assess the shelf life of the pesticide formulation. At the same time, the contact angle of the pesticide formulation on paraffin and its interfacial tension were also determined for the spraying application. In addition, the insecticidal activity of bifenthrin nanoemulsions against cabbage maggots was further studied. To the best of our knowledge, the formation of biodiesel nanoemulsions for bifenthrin formulation by the PIC method has rarely been reported so far. The main objective of this work is to obtain the optimum emulsification conditions for the preparation of transparent biodiesel nanoemulsions via the PIC method, and to evaluate the long-term stability of the bifenthrin nanoemulsions for the assessment of the shelf life of the pesticide formulation.

\section{Experimental procedures}

\subsection{Materials}

Biodiesel (major component: fatty acid methyl) and nonylphenol polyoxyethylene ether (NP-6, the average number of ethylene oxide groups was 6) were purchased from Shanghai Weipu Chemicals Co. Ltd. (Shanghai, China). Bifenthrin (standard substance) and calcium dodecylbenzene sulfonate (ABSCa) were provided by Aladdin Chemical Reagent Co. Ltd. (Shanghai, China). The chemicals were of analytical grade and were used without further purification. Double distilled water was used in the preparation of all solutions and nanoemulsions.

\subsection{Methods}

2.2.1 Preparation of bifenthrin nanoemulsions. The preparation of nanoemulsions with a bifenthrin concentration of 8 wt\% was achieved by the PIC method. ${ }^{23}$ The low-energy method was performed by the stepwise addition of water to the mixture of oil (biodiesel) and the mixed surfactants (NP-6 and ABSCa) at $25{ }^{\circ} \mathrm{C}$. Firstly, bifenthrin (8 wt\%) was fully dissolved in a certain amount of biodiesel, and then the drugloaded biodiesel was blended with the mixed surfactants to form the mixture as the oil phase. The bifenthrin nanoemulsions were formed by adding the water to the oil phase, with magnetic stirring. The interactions between bifenthrin and bifenthrin nanoemulsions were respectively characterized by FT-IR and UV-vis spectroscopy. FT-IR measurements were performed on a Tensor 27 Fourier transform infrared spectrometer (Bruker, Germany). UV-vis spectroscopic experiments were implemented by dissolving the composite materials in 
$0.1 \mathrm{~mol} \mathrm{~L}^{-1} \mathrm{pH}$ 7.0 PBS, and using a Cary 50 probe spectrophotometer (Varian, Australia).

2.2.2 Pseudo-ternary phase diagrams. The pseudo-ternary phase diagram was obtained at $25^{\circ} \mathrm{C}$ by the stepwise addition of water to the mixture of the oil and the mixed ionic-nonionic surfactants. The mass ratio of NP- 6 and ABSCa was fixed at 5/5, and the water addition rate was fixed at $0.5 \mathrm{~mL} \mathrm{~min}^{-1}$. After each addition, the samples were stirred and homogenized with a vibromixer at a speed of $8000 \mathrm{rpm}$. The phase separation was observed by the naked eye. Changes in the appearance of the sample (transparent-to-turbidity or turbidity-to-transparent transitions) were recorded as the phase boundary. ${ }^{24}$ Three parallel experiments were carried out for every sample and the mean value was taken. Each sample was homogenized with a vibromixer and kept at $25{ }^{\circ} \mathrm{C}$ to equilibrate. Optically anisotropic liquid crystalline phases were identified by using polarizing light microscopy (PLM, DMRX, LEICA, Germany) through the identification of characteristic textures. The composition of the sample was calculated from the weight measurement of all components. The boundary lines drawn on the phase diagram were equidistant between consecutive experimental measurements on either side of the phase boundary. ${ }^{\mathbf{8} 20}$

2.2.3 Transmission electron microscopy (TEM). The morphologies of the nanoemulsion droplets were observed using a JEM 2100 TEM (JEOL Co., Japan) at an acceleration voltage of $200 \mathrm{kV}$. TEM images of the samples were obtained by placing a few drops of the nanoemulsion on a carbon-coated copper grid, negative staining with $2 \%$ phosphotungstic acid and evaporation prior to observation.

2.2.4 Determination of nanoemulsion droplet size. The mean droplet size and size distribution of the nanoemulsions were determined by dynamic light scattering (DLS) with a Malvern Nano-ZS90 Zetasizer (UK) at a scattering angle of $90^{\circ}$ at $25{ }^{\circ} \mathrm{C}$, employing an (He-Ne) argon laser $(\lambda=633 \mathrm{~nm})$. Prior to the measurement, the samples were diluted $1 / 100$ with double distilled water to avoid multiple scattering.

2.2.5 Contact angle and interfacial tension measurement. The contact angle measurements were conducted on a HARKE Contact Angle Meter equipped with a color CCD camera (Beijing, China). About $10 \mu \mathrm{L}$ of nanoemulsion was dropped on the solid paraffin sheet. The contact angles of nanoemulsions were obtained according to the shape of the drop by calculating the slope of the tangent to the drop at the liquid-solid-vapor (LSV) interface line.

Equilibrium interfacial tension was measured at $25^{\circ} \mathrm{C}$ with a Sigma 70 tensiometer employing the DuNoüy ring method (Bruker, Germany). The average value of three measurements was used.

2.2.6 Insecticidal activity of bifenthrin nanoemulsions against cabbage maggots. In order to evaluate the practical application of the pesticide formulation, actual pesticide testing using biological and botanical studies were performed by measuring the insecticidal activity of bifenthrin nanoemulsions against cabbage maggots. The cabbage maggots collected from field-grown broccoli plants in Hainan, China, were treated with different dilution ratios of bifenthrin nanoemulsions according to the previous method. ${ }^{25}$ Forty cabbage maggots were placed in a $250 \mathrm{~mL}$ sterile beaker containing $100 \mathrm{~mL}$ of the diluted bifenthrin nanoemulsions. All the samples were maintained at room temperature. The insecticidal activity of the bifenthrin nanoemulsions was monitored by recording the mortality after 5, 10 and 20 h of exposure. Dead cabbage maggots were identified when they failed to move. Each test was performed in triplicate and the mean value was taken.

2.2.7 Bifenthrin analysis by high-performance liquid chromatography (HPLC). To further probe the long-term stability of the bifenthrin formulation, bifenthrin was quantified using HPLC after being stored for three months. The chromatographic system consisted of a UV detector set at $225 \mathrm{~nm}$ and connected to a Waters 1500 Series HPLC pump equipped with a $5 \mathrm{~mm} \times 150 \mathrm{~mm} \times 4.6 \mathrm{~mm}$ Spherisorn ODS column. Bifenthrin analysis was performed at room temperature with an 85 : 15 methanol : water $(\mathrm{v} / \mathrm{v})$ mobile phase; $5.0 \mu \mathrm{L}$ of sample was injected at a flux rate of $1 \mathrm{~mL} \mathrm{~min}^{-1}$, and the retention time was about $5.4 \mathrm{~min}$.

\section{Results and discussion}

\subsection{Nanoemulsions formation by PIC method}

At constant pressure and temperature, the phase behavior of a three-component system can be represented by a planar triangle, called a ternary phase diagram. This is the most convenient and effective way to study the phase, composition and phase boundary of the equilibrium system. According to the phase diagram, the structural state of the mixed system can be preliminarily estimated, which can intuitively reflect the change in the phase state of the microemulsion system. ${ }^{\mathbf{1 3}}$ Through the drawing of the pseudo-ternary phase diagram, the compatibility of the substances in the system and the conditions for forming the microemulsions can be studied. ${ }^{8}$ The pseudo-ternary phase diagram for the equilibrium phase behavior of biodiesel, water and the mixed ionic-nonionic surfactants was drawn with the aid of the naked eye and polarizing light microscopy. As shown in Fig. 2, the pseudoternary phase diagram of biodiesel, water and the mixed ionic-nonionic surfactants was divided into six different areas. These areas included, respectively, an inverse micellar solution of a W/O microemulsion (Om), a lower liquid crystalline phase with an upper colorless liquid phase $(\mathrm{L} \alpha+\mathrm{Om})$, a lamellar liquid crystalline phase $(\mathrm{L} \alpha)$, a bicontinuous microemulsion (D), a micellar solution of an $\mathrm{O} / \mathrm{W}$ microemulsion $(\mathrm{Wm})$ and a multiphase region $(\mathrm{M})$. The $\mathrm{Om}, \mathrm{D}$ and $\mathrm{Wm}$ phases were isotropic, colorless fluids, but the others contained several twoand multiple-phase regions. ${ }^{8}$

Under the guidance of the pseudo-ternary phase diagram, we could accurately prepare the drug-loaded nanoemulsion by the PIC method, which involved a combination of phase-inversion and self-emulsification. The procedure to obtain nanoemulsions by the PIC method consisted of progressively adding one of the components (water or oil) to a mixture of the other two components (oil-surfactant or water-surfactant, respectively).$^{\mathbf{1 3 , 2 6 , 2 7}}$ In our work, the preparation of the nanoemulsions was achieved by dripping the water phase into the oil-surfactant phase. For example, we fixed the mass ratio of biodiesel and the mixed surfactants $\left(\mathrm{mR}_{\mathrm{OS}}\right)$ at 1.4. Along with the addition of 


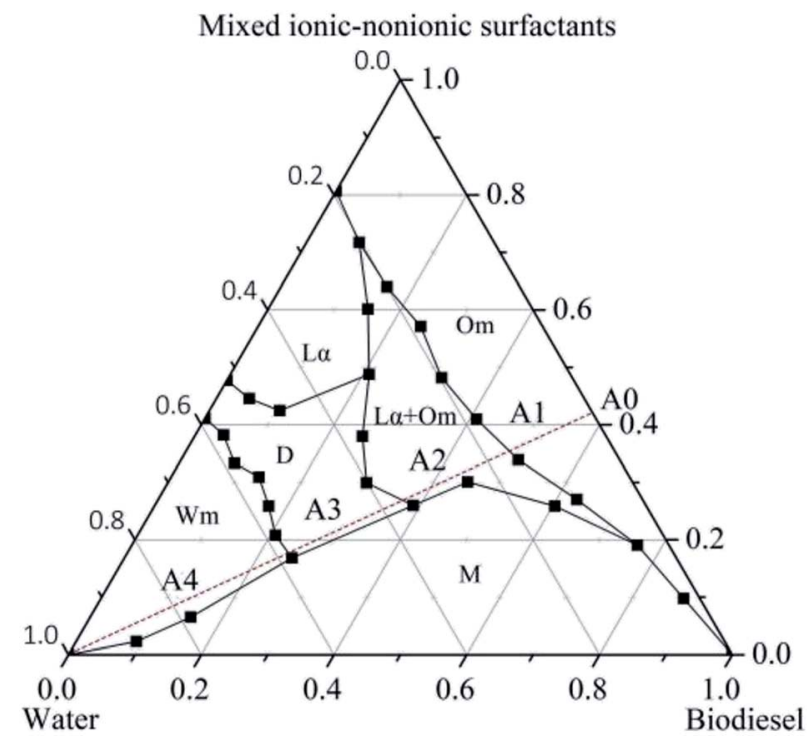

Fig. 2 Pseudo-ternary phase diagram of biodiesel, water and the mixed ionic-nonionic surfactants system at $25^{\circ} \mathrm{C}$.

water, the preparation procedure of the nanoemulsions was gradually performed according to the A0-A4 route, as shown in Fig. 2. Firstly, a certain amount of biodiesel dissolving bifenthrin was blended with the mixed surfactants to form the mixture (A0 system). When water was dripped progressively into the oil phase, the initial system generally formed a W/O microemulsion (A1 system). Then, as the mass fraction of water increased, water droplets merged together to form a blend of a lamellar liquid crystalline phase and W/O microemulsion (A2). Subsequently, with the continued increase in the mass fraction of water, the hydration grade of the mixed ionicnonionic surfactants was progressively enhanced, and the spontaneous curvature of the mixed surfactants changed from negative to zero, thus resulting in the formation of a bicontinuous microemulsion (A3). ${ }^{13}$ The mixed ionic-nonionic surfactant layer had higher flexibility to partition into the oil-water interface and decreased the oil-water interfacial tension instantaneously. ${ }^{28-31}$ Upon further increasing the water content, the bicontinuous microemulsion with zero curvature would generate phase inversion and decompose into a very finely dispersed $\mathrm{O} / \mathrm{W}$ microemulsion (A4), which contained a very high positive curvature of the surfactant layer..$^{32}$ The mechanism of the formation of bifenthrin nanoemulsions by dripping the water phase into the oil-surfactant phase was exemplified in Fig. 3, which was consistent with the previous report. ${ }^{24}$

The resultant bifenthrin nanoemulsions were observed to be light blue and transparent, which exhibited low viscosity and good stability, as shown in Fig. 4a. The mean droplet size of the nanoemulsions and the morphology of the nanoemulsion droplets were respectively evaluated by DLS and TEM measurements. As shown in Fig. 4b, the obtained bifenthrin nanoemulsions possessed a narrow size distribution ranging from $45 \mathrm{~nm}$ to $90 \mathrm{~nm}$, and their mean size was $63.8 \mathrm{~nm}$ with the PDI of 0.101. Moreover, TEM images (Fig. 4c and d) revealed that the bifenthrin nanoemulsions exhibited a similar spherical shape with the mean size of $62.3 \mathrm{~nm}$, which was similar to the results of DLS measurements. Due to the coverage of the mixed ionic-nonionic surfactants at the oil-water interface, which protected the emulsion droplets against coalescence, ${ }^{33}$ the formed $\mathrm{O} / \mathrm{W}$ nanoemulsions could show a very fine dispersion with small and uniform emulsion droplets, thus exhibiting good stability.

The biodiesel used in this work was obtained from soybean oil by chemical reaction with an alcohol, and its main component is fatty acid methyl ester. Since the chemical configuration of biodiesel may influence the preparation and stability of the nanoemulsion, we conducted FT-IR and UV-vis spectroscopy studies to characterize the structure of pure bifenthrin and the mixture of bifenthrin nanoemulsions. As shown in Fig. 5a, the characteristic peaks of bifenthrin at $3069.62,1587.63,1487.20$ and $812.65 \mathrm{~cm}^{-1}$ were respectively assigned to $\mathrm{C}-\mathrm{H}$ stretching vibrations and skeleton vibrations of the benzene ring. ${ }^{34}$ The peaks at 2966.41 and $1725.79 \mathrm{~cm}^{-1}$ were respectively attributed to symmetric stretching vibrations of $\mathrm{C}-\mathrm{H}$ for methyl and $-\mathrm{C}=\mathrm{O}$ for ester groups. ${ }^{35}$ In comparison with bifenthrin, the bifenthrin nanoemulsion exhibited a different FT-IR spectrum. The peak at $3048.84 \mathrm{~cm}^{-1}$ was ascribed to the stretching vibration of the unsaturated double bond in the fatty acid methyl ester carbon skeleton. The peaks at 1731.13 and $1189.77 \mathrm{~cm}^{-1}$ were respectively assigned to symmetric stretching vibrations of $-\mathrm{C}=\mathrm{O}$ and the characteristic absorption peak of fatty acid methyl ester. The above results implied that the bifenthrin was effectively encapsulated in the micelle structure of the mixed surfactant during the preparation of bifenthrin nanoemulsions. It was the coverage of the mixed surfactant that reduced the UV-vis absorbance of bifenthrin in the nanoemulsions, as shown in Fig. $5 b$.

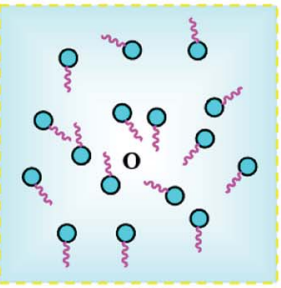

(A0)

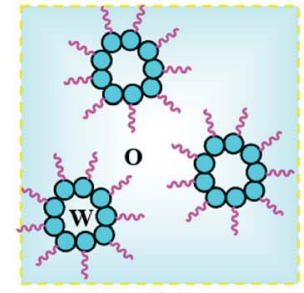

(A1)

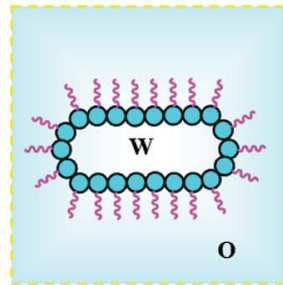

(A2)

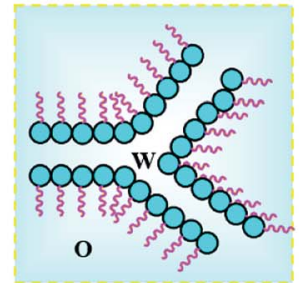

(A3)

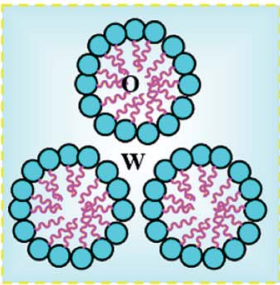

(A4)

Hydrophilic head group $\quad \sim \Omega$ Hydrophobic tail group

Fig. 3 Schematic description of the formation mechanism of bifenthrin nanoemulsions by dripping the water phase into the oil-surfactant phase. 
(a)

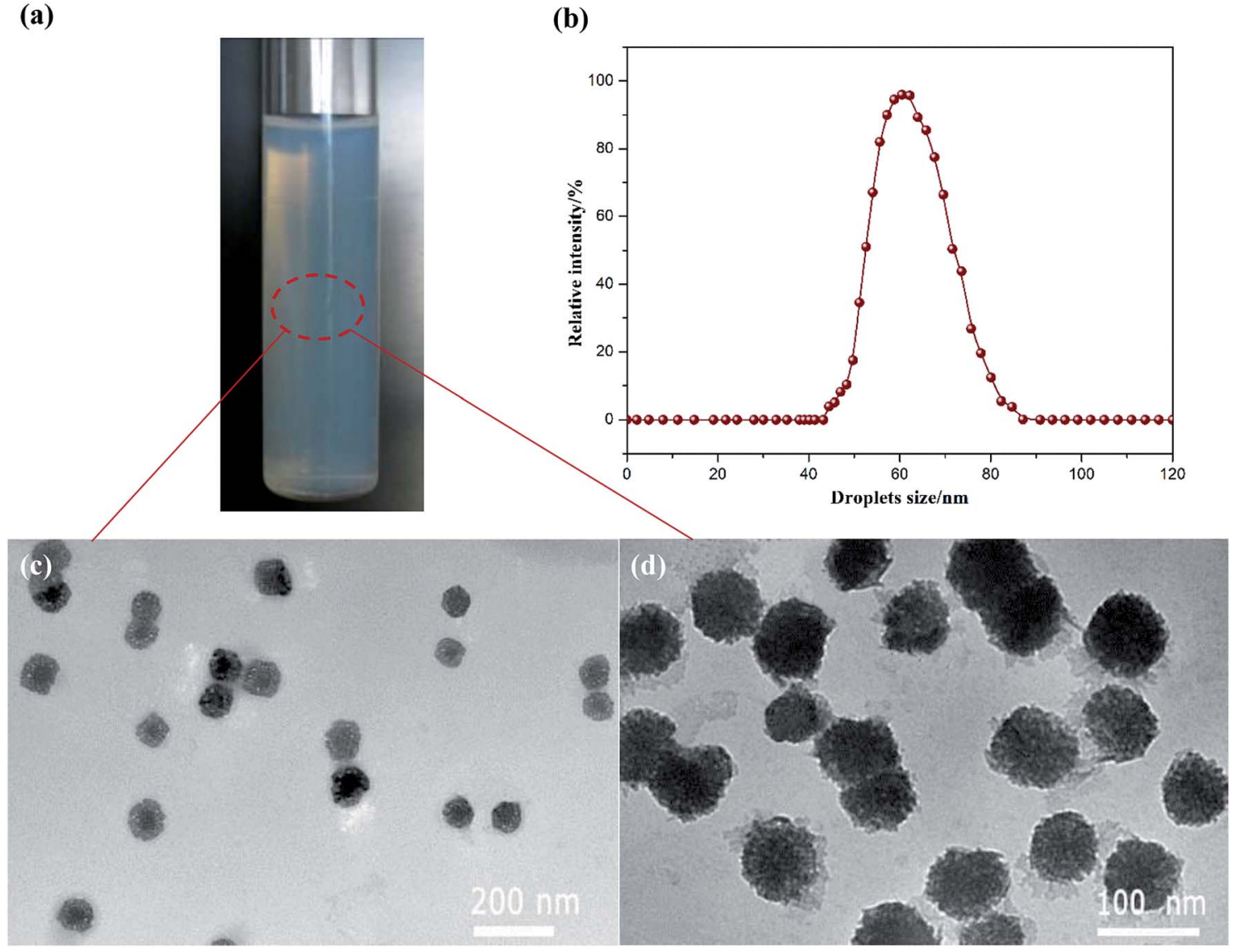

Fig. 4 (a) An optical image of bifenthrin nanoemulsions. (b) Size distribution of bifenthrin nanoemulsions measured by DLS. (c and d) TEM images of the dispersion of nanoparticles as obtained after evaporation of the solvent of the bifenthrin nanoemulsions.

\subsection{Effect of the emulsification conditions on the mean droplet size of the bifenthrin nanoemulsion}

Various previous reports that used the PIC method to prepare the nanoemulsions have shown that the droplet size generally depends on many emulsification conditions, including the mass ratio of NP- 6 and $\mathrm{ABSCa}, \mathrm{mR}_{\mathrm{OS}}$, stirring rate, the water addition rate and the emulsification temperature. ${ }^{36-38}$ As the relationship between them was very complicated, other experimental conditions were kept unchanged during the experiment, and one of the factors was changed to test its effect on the mean droplet size of the bifenthrin nanoemulsion. (a)

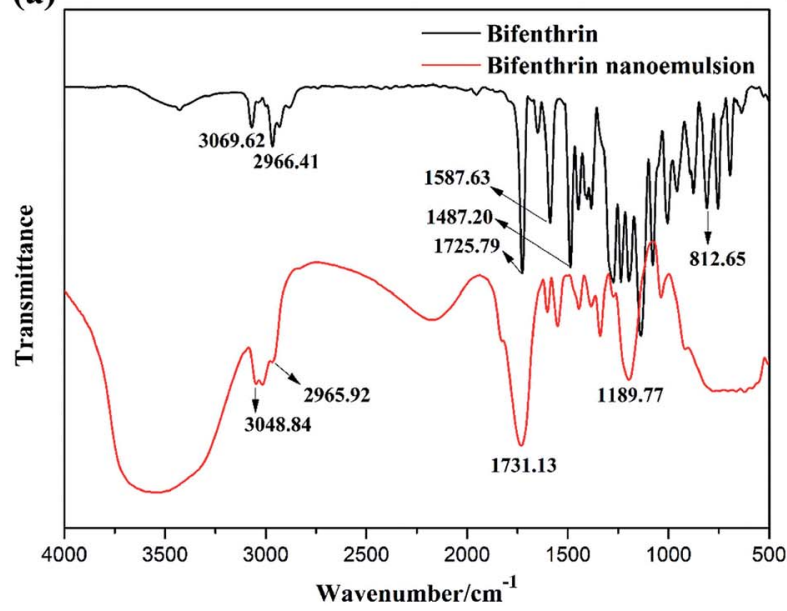

(b)

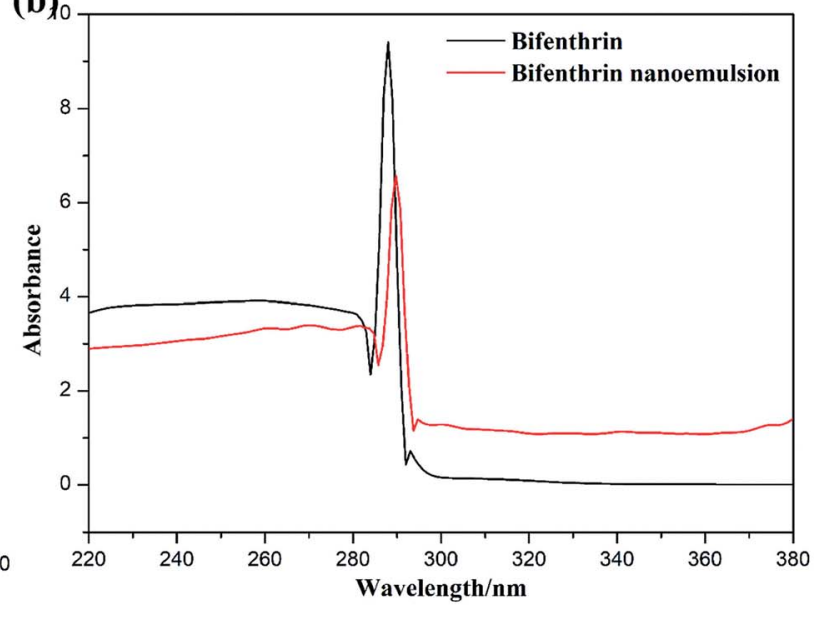

Fig. 5 (a) FT-IR and (b) UV-vis absorption spectra of bifenthrin and bifenthrin nanoemulsions. 
3.2.1 Effect of the mass ratio of NP-6 and ABSCa on the mean droplet size of the nanoemulsion. The effect of the mass ratio of NP-6 and ABSCa on the mean droplet size of bifenthrin nanoemulsion is shown in Fig. 6a. With the decrease in the mass ratio of NP-6 and ABSCa, the mean droplet size of bifenthrin nanoemulsion first decreased rapidly and then increased. The results were attributed to the fact that the mixed anionicnonionic micelles in the solution mainly depended on the steric repulsion between the nonpolar heads of the nonionic surfactant and the electrostatic repulsion of the anionic head groups. ${ }^{39}$ When anionic surfactants were added to the nonionic surfactant micelles, the distance between the nonpolar heads of the ethoxy groups increased. With the increase of the anionic surfactant, the steric repulsion decreased, and the mixed surfactant molecules in the emulsion particles were closer, leading to the smaller droplets size; therefore, the mean droplet size of the bifenthrin nanoemulsion firstly decreased with the decrease of the mass ratio of NP-6 and ABSCa. However, excessive sulfonic acid groups would also produce relatively obvious electrostatic repulsion, resulting in loose emulsion particles, thus increasing the droplet size. Therefore, when the anionic surfactant exceeded a certain amount, the mean droplet size of nanoemulsion increased with the further increase of anionic surfactant, i.e., with the further decrease of the mass ratio of NP-6 and ABSCa, the mean droplet size of bifenthrin nanoemulsion would increase. Consequently, the optimum mass ratio of NP- 6 and ABSCa was fixed at 5/5.

3.2.2 Effect of $\mathrm{mR}_{\mathrm{OS}}$ on the mean droplet size of the nanoemulsion. The $\mathrm{mR}_{\mathrm{OS}}$ is an important index to measure the emulsifying properties of the bifenthrin nanoemulsion. The higher the $\mathrm{mR}_{\mathrm{OS}}$ was, the stronger the emulsification performance of the system was, thus enhancing the ability to solubilize the oil phase, enlarging the drug-loading rate, and improving the application value. The effect of $\mathrm{mR}_{\mathrm{OS}}$ on the mean droplet size of the bifenthrin nanoemulsion is shown in Fig. 6b. It was obvious that the mean droplet size of the bifenthrin nanoemulsion firstly decreased as the $\mathrm{mR}_{\mathrm{OS}}$ increased, and then increased as the $\mathrm{mR}_{\mathrm{OS}}$ further increased. When the $\mathrm{mR}_{\mathrm{OS}}$ gradually increased, the mass fraction of the mixed surfactant gradually decreased. Low $\mathrm{mR}_{\mathrm{OS}}$ possibly produced the separation of a lamellar liquid crystalline phase, which led to the higher droplet size. ${ }^{8}$ For low surfactant concentrations, the oil solubilization was not complete. So the high $\mathrm{mR}_{\mathrm{OS}}$ caused the separation of an excess oil phase, also resulting in the higher droplet size. ${ }^{33}$ On the basis of the above results, the optimum $\mathrm{mR}_{\mathrm{OS}}$ for nanoemulsion formation was 1.4.

3.2.3 Effect of the stirring rate on the mean droplet size of the nanoemulsion. Stirring dispersed the added water so that the components of the emulsion could be uniformly mixed, which had a great influence on the nanoemulsion formation.
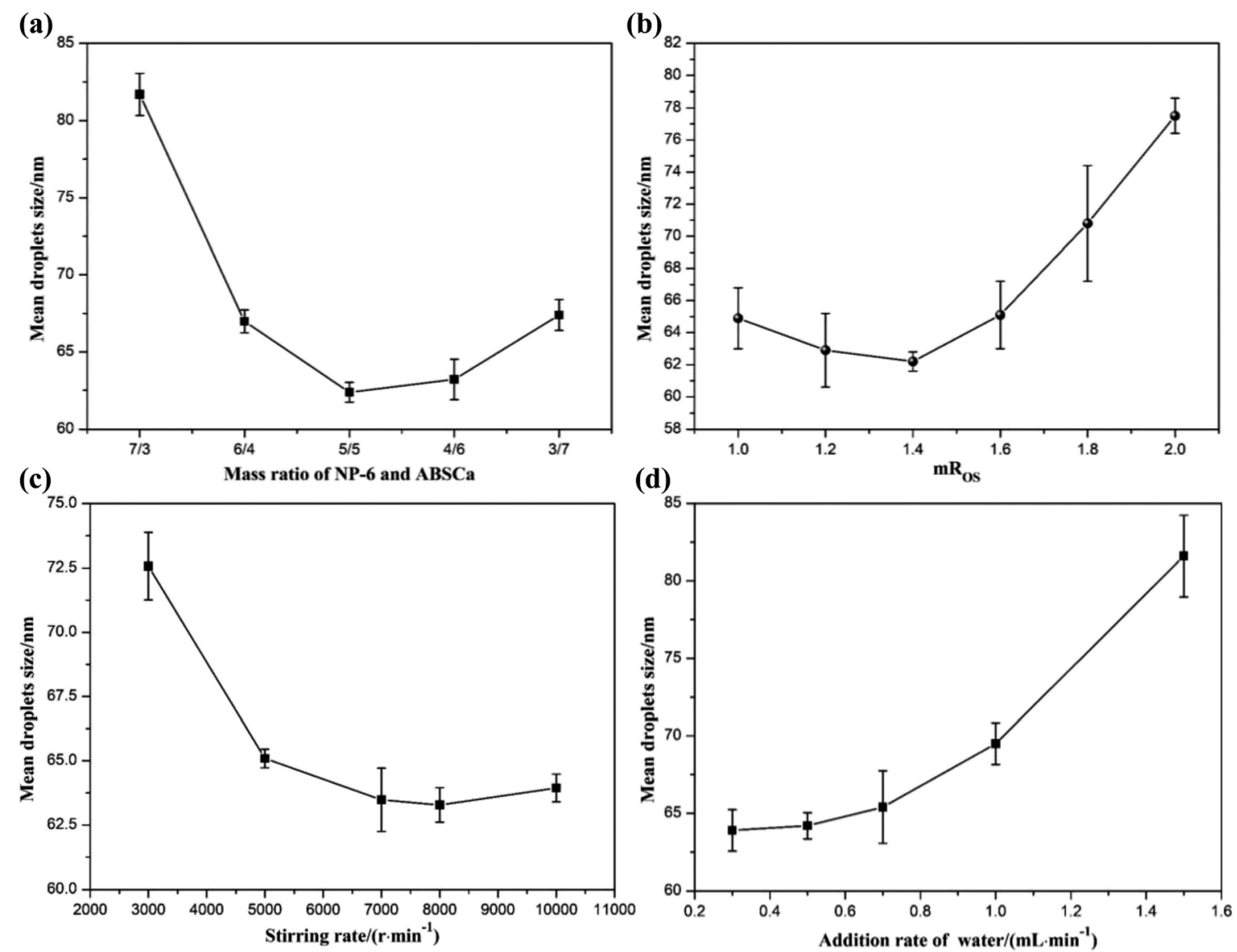

Fig. 6 Effects of (a) the mass ratio of NP-6 and ABSCa, (b) $\mathrm{mR}_{\mathrm{OS}}$ (c) stirring rate and (d) the addition rate of water on the mean droplet size of bifenthrin nanoemulsions produced by the PIC method. 
The stirring rate is an important factor affecting the mean droplet size of the bifenthrin nanoemulsion. As shown in Fig. 6c, as the stirring rate increased, the mean droplet size of bifenthrin nanoemulsion firstly decreased rapidly and then tended to the plateau value. When the stirring rate was less than $7000 \mathrm{rpm}$, the magnetic force of the stirring was insufficient to uniformly mix the emulsion, and it uniformly dispersed the dripping water, resulting in the uneven distribution of the surfactant layer, weak hydration and larger droplet size during the formation of the nanoemulsion. When the stirring rate was in the range of 7000-8000 rpm, the mean droplet size of the bifenthrin nanoemulsion was the smallest. When the stirring rate was greater than $8000 \mathrm{rpm}$, ascribed to the fast stirring rate, the system with higher viscosity was difficult to be effectively dispersed, thus resulting in a slight increase in droplets size. Therefore, the optimum stirring rate for nanoemulsion formation was set at $8000 \mathrm{rpm}$.

3.2.4 Effect of the addition rate of water on the mean droplet size of the nanoemulsion. During the nanoemulsion formation, the hydration of the nonionic surfactant, the polyoxyethylene chain (PEO chain), was enhanced with the increase of water mass fraction, and phase-inversion occurred to form an $\mathrm{O} / \mathrm{W}$ nanoemulsion. The addition rate of water would affect the speed of hydration of nonionic surfactant, and an excessively fast addition rate of water would lead to local hydration being stronger than other regions, resulting in the uneven distribution of the nanoemulsion droplet size and affecting the mean droplet size of the nanoemulsion. The effect of the addition rate of water on the mean droplet size of bifenthrin nanoemulsion is shown in Fig. 6d. When the addition rate of water was less than

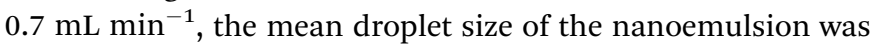
very small. Since the water droplets that were dripped into the emulsion were well dispersed, they had little effect on the formation of the nanoemulsion and its mean droplet size. When the addition rate of water exceeded $0.7 \mathrm{~mL} \mathrm{~min}^{-1}$, the mean droplet size of the nanoemulsion increased faster and faster with the increase of the addition rate of water. As the stirring was insufficient to uniformly disperse the added water, the hydration strength of the surfactant layer in the emulsion was not uniform, thus resulting in the generation of large-sized droplets. As such, the optimum addition rate of water for nanoemulsion formation was set as $0.7 \mathrm{~mL} \mathrm{~min}{ }^{-1}$.

3.2.5 Effect of the emulsification temperature on the mean droplet size of the nanoemulsion. Since the hydrophilic and lipophilic properties of nonionic surfactants are easily affected by the emulsification temperature, they can have an effect on the mean droplet size of the nanoemulsion. ${ }^{40}$ Therefore, the mixed surface can be indirectly examined by investigating the effect of temperature on the average particle size of the formed nanoemulsion. The interaction between the components of the mixed ionic-nonionic surfactants could be indirectly evaluated by investigating the effect of the emulsification temperature on the mean droplet size of the formed nanoemulsion. The mean droplet size of the nanoemulsion as a function of the emulsification temperature is shown in Fig. 7, where it can be observed that the emulsification temperature had little effect on the mean droplet size of the nanoemulsion, and the mean droplet

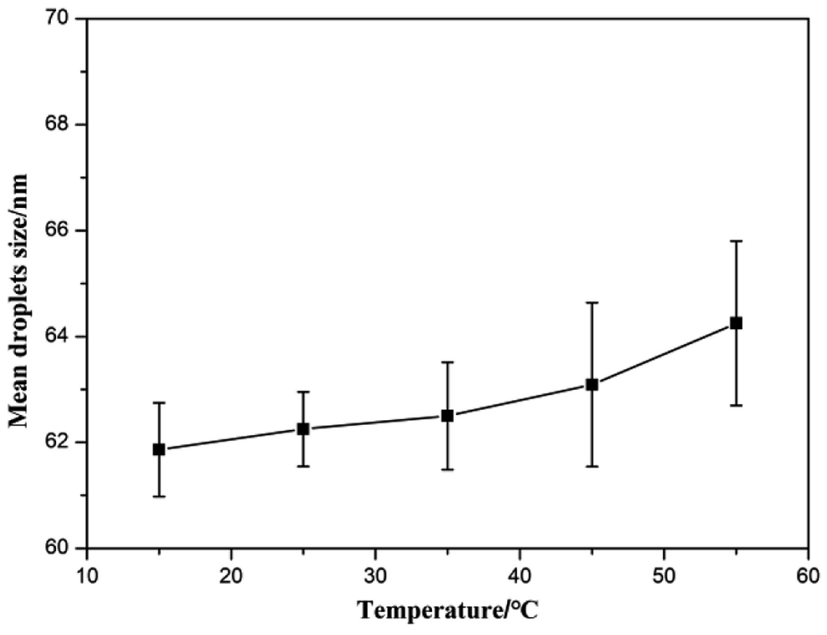

Fig. 7 Effect of the emulsification temperature on the mean droplet size of bifenthrin nanoemulsions.

size of the nanoemulsion increased slightly with the increase of the emulsification temperature. The results were ascribed to the fact that the incorporation of an ionic surfactant could significantly increase the turbidity point of the single nonionic surfactant, which improved the hydration of nonionic surfactants against the emulsification temperature. Thus, the amount of mixed ionic-nonionic surfactant molecules adsorbed at the $\mathrm{O} / \mathrm{W}$ interface avoided the influence of the emulsification temperature on the emulsion size. Based on the above results, the emulsification temperature for nanoemulsion formation in our work was fixed at $25{ }^{\circ} \mathrm{C}$.

\subsection{Interfacial tension and contact angle of the bifenthrin nanoemulsion}

Nanoemulsions have been shown to be advantageous for optimizing the delivery of hydrophobic compounds in agrochemicals. The small droplets of nanoemulsions allow them to deposit uniformly on plant leaves. ${ }^{8}$ Wetting, spreading and penetration would be also enhanced due to the low interfacial tension of the $\mathrm{O} / \mathrm{W}$ nanoemulsions. ${ }^{41}$ As shown in Fig. 8, with

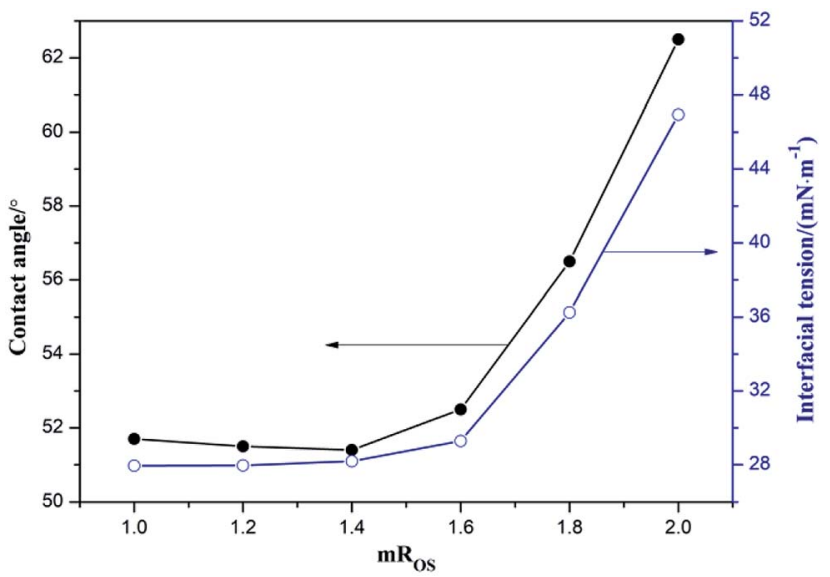

Fig. 8 Variation curves of interfacial tension and the contact angle of bifenthrin nanoemulsion at different $\mathrm{mR}_{\mathrm{O}}$. 
the increase of $\mathrm{mR}_{\mathrm{OS}}$, the interfacial tension of the bifenthrin nanoemulsion gradually increased. When the $\mathrm{mR}_{\mathrm{OS}}$ did not exceed 1.6, the interfacial tension was less than $29 \mathrm{mN} \mathrm{m}^{-1}$, without significant change. In this case, the bifenthrin nanoemulsion had low interfacial tension, good emulsifying performance, and easy adsorption on the plant surface, thus effectively improving the utilization of bifenthrin. The smaller the $\mathrm{mR}_{\mathrm{OS}}$ in the nanoemulsion system, the larger was the mixed surfactant mass fraction, and the mixed surfactant could effectively reduce the interfacial tension of the bifenthrin nanoemulsion. Therefore when the $\mathrm{mR}_{\mathrm{OS}}$ was less than 1.6, the interfacial tension was small, and the bifenthrin nanoemulsion could be suitable for spraying application. In addition, the effects of pesticide nanoemulsion on wetting, spreading and leaf penetration are also commonly expressed by the contact angle.

The leaves of the plants had a layer of wax that was highly hydrophobic. Therefore, the use of paraffin wax instead of plant leaves to measure the contact angle could similarly characterize the wetting of bifenthrin nanoemulsion on the plant leaves. As shown in Fig. 9, the contact angle of the double distilled water on the paraffin surface was $101^{\circ}$, which was non-wetting, while the contact angle of the bifenthrin nanoemulsion on the paraffin surface was obviously less than $90^{\circ}$, indicating that it was partially or totally wet. In addition, the droplet shapes of the double distilled water and the bifenthrin nanoemulsion on the real plant leaves were also measured. As shown in Fig. 10, the bifenthrin nanoemulsion droplets could be effectively spread on the real plant leaves in comparison with the double distilled water droplets, indicating good wetting properties, which is in good agreement with the results on the paraffin. These results imply that the bifenthrin nanoemulsion could be spread on the leaf surface, which indicated the potential for agrochemical active delivery. It could be also seen from Fig. 6 that the contact angle of the bifenthrin nanoemulsion was basically increased with the increase of the $\mathrm{mR}_{\mathrm{OS}}$, when the $\mathrm{mR}_{\mathrm{OS}}$ exceeded 1.6. Since the higher $\mathrm{mR}_{\mathrm{OS}}$ led to the lower mass fraction of the mixed surfactant, the thinner surfactant layer in the emulsion resulted in a worse wetting effect. Therefore, the optimum $\mathrm{mR}_{\mathrm{OS}}$ for spraying application was 1.4 , which was in accordance with the $\mathrm{mR}_{\mathrm{OS}}$ for nanoemulsion formation.

\subsection{Insecticidal activity against cabbage maggots}

The insecticidal activity of bifenthrin nanoemulsions was studied against cabbage maggots after dilution. Table 1 shows the mortality of cabbage maggots over time, after 100-fold, 500fold and 1000-fold dilution of bifenthrin nanoemulsions. The mortality rate of cabbage maggots increased with the increase in exposure time from $5 \mathrm{~h}$ to $20 \mathrm{~h}$. It is worth noting that after $20 \mathrm{~h}$ of exposure, the mortality rate for all samples exceeded $90 \%$, and $100 \%$ mortality was observed at 100 -fold and 500 -fold dilution of bifenthrin nanoemulsions with the exposure time of $20 \mathrm{~h}$. These results indicate the high insecticidal activity of bifenthrin nanoemulsion for practical application.

\subsection{Stability of the bifenthrin nanoemulsion}

The formed nanoemulsions exhibited good stability without phase separation after several days, but an increase in droplet size was observed with time. There are two probable breakdown processes in dispersed systems, namely, coalescence and Ostwald ripening. The droplet growth rate, $\omega$, due to Ostwald ripening, can be calculated by the Lifshitz-Slezov and Wagner (LSW) theory: ${ }^{5,42,43}$

$$
\omega=\frac{\mathrm{d} r^{3}}{\mathrm{~d} t}=\frac{8 C_{\infty} \gamma V_{\mathrm{m}} D}{9 \rho R T}
$$

where $r$ is the average droplet radius after time $t ; D$ is the diffusion coefficient of the oil in the continuous phase; $V_{\mathrm{m}}$ is the molar volume of the oil; $\gamma$ is the interfacial tension; $C_{\infty}$ is the bulk phase solubility (the solubility of the oil in an infinitely large droplet); $R$ is the gas constant; $\rho$ is the density of the oil; $T$ is the absolute temperature.

In order to verify that the breakdown process was Ostwald Ripening, the cube of the radius, $r^{3}$, was plotted as a function of time at different $\mathrm{mR}_{\mathrm{OS}}$ in Fig. 11. The linear variation of $r^{3}$ as a function of time indicated that Ostwald ripening was the driving force for instability, which was similar to the results obtained by Du et al. ${ }^{44}$ It was clearly illustrated that the slope increased with the increase in $\mathrm{mR}_{\mathrm{OS}}$, which meant that the more stable nanoemulsions were formed with higher surfactant concentrations. These results were in agreement with previous research results. ${ }^{6,8}$ Note that the interfacial tension was reduced with the increase in surfactant concentration, which is a factor for reducing the Ostwald ripening, according to in eqn (1).
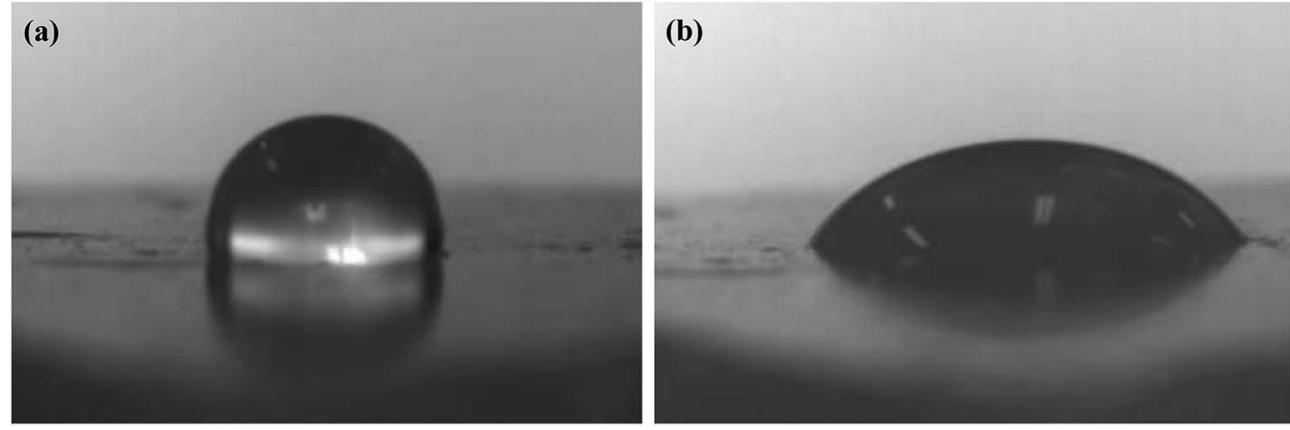

Fig. 9 Droplet shape of (a) bifenthrin nanoemulsions and (b) double distilled water on paraffin surface. 

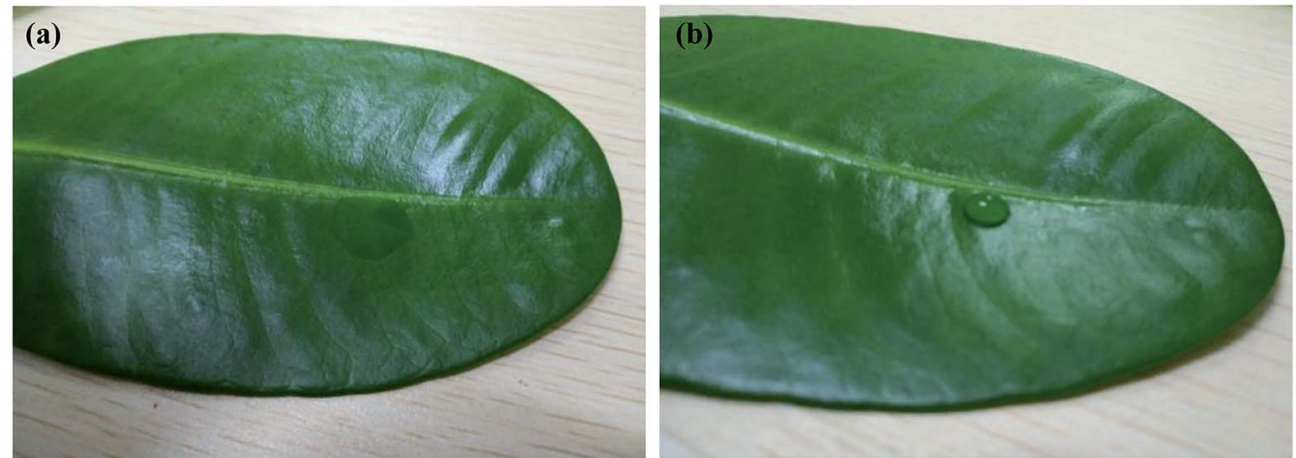

Fig. 10 Droplet shape of (a) bifenthrin nanoemulsions and (b) double distilled water on the real plant leaves.

Table 1 Mortality of cabbage maggots at various dilution ratios of bifenthrin nanoemulsions

\begin{tabular}{llll}
\hline & \multicolumn{3}{l}{ Mortality/\% } \\
\cline { 2 - 4 } Dilution ratios & $5 \mathrm{~h}$ & $10 \mathrm{~h}$ & $20 \mathrm{~h}$ \\
\hline 100 -fold & $72.5 \pm 5.0^{a}$ & $100 \pm 0$ & $100 \pm 0$ \\
500 -fold & $52.5 \pm 2.5$ & $77.5 \pm 5.0$ & $100 \pm 0$ \\
1000 -fold & $37.0 \pm 2.5$ & $62.5 \pm 2.5$ & $92.5 \pm 5.0$ \\
& & \\
${ }^{a}$ Values represent the standard deviation.
\end{tabular}

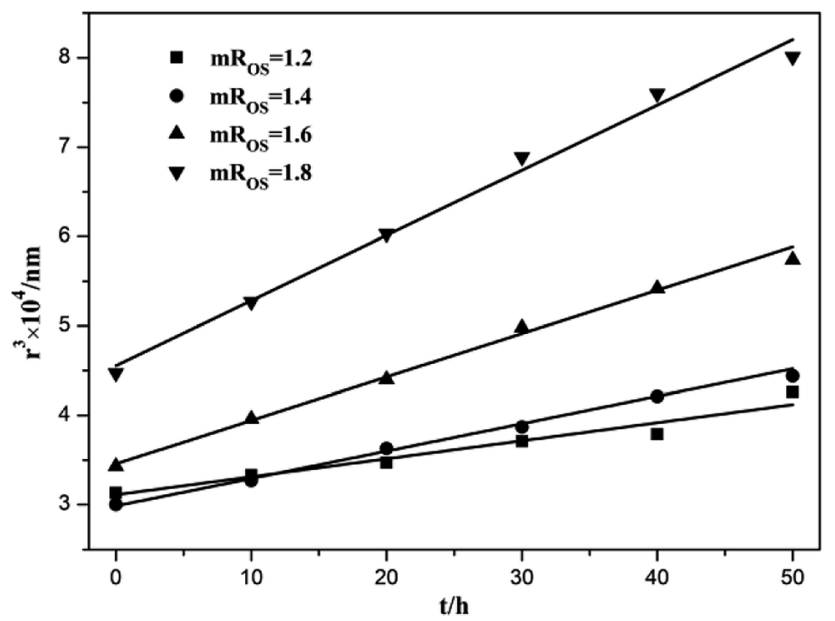

Fig. 11 The cube of the radius versus time for bifenthrin nanoemulsions at various $\mathrm{mRos}_{\text {. }}$

The long-term stability of the bifenthrin nanoemulsion was further probed to assess the shelf life of the bifenthrin formulation. The chemical stability was represented by the amount of bifenthrin retention measured by HPLC analysis at the storage time of three months. Fig. 12 shows the representative chromatogram of the bifenthrin nanoemulsion. The peaks at the retention time of $3.3 \mathrm{~min}$ were assigned to the biodiesel, while the retention time of bifenthrin was about $5.4 \mathrm{~min}$. It could be seen that after three months of storage, the peak intensity was slightly weakened, revealing that the bifenthrin nanoemulsion

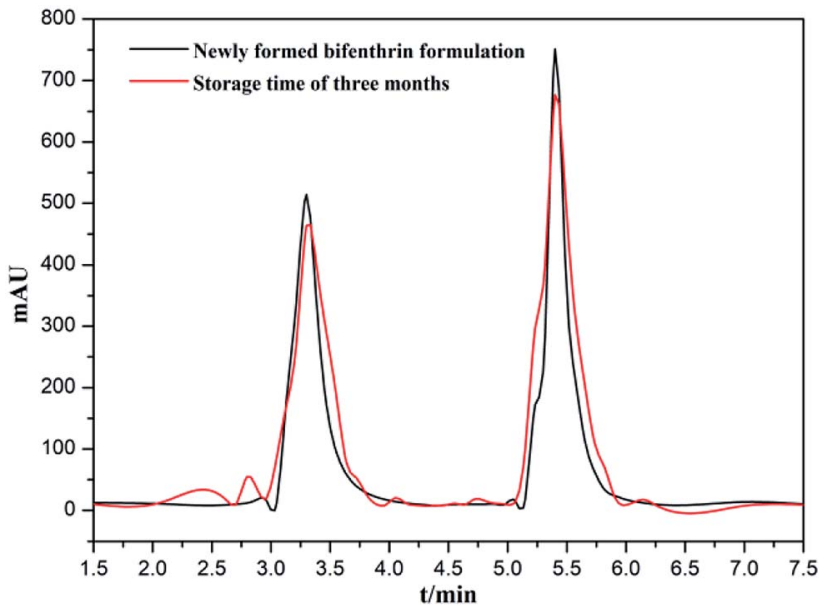

Fig. 12 Representative chromatogram of bifenthrin nanoemulsions by HPLC analysis.

is not easily decomposed or precipitated, and has a good shelf life, making it suitable for potential pesticide formulations.

\section{Conclusion}

A stable $\mathrm{O} / \mathrm{W}$ bifenthrin nanoemulsion was successfully prepared by the PIC method, using NP-6 and ABSCa as the mixed surfactants, and biodiesel as the oil phase. The mechanism of the formation of bifenthrin nanoemulsions by dripping the water phase into the oil-surfactant phase was stated with the aid of the pseudo-ternary phase diagram. To obtain the optimum emulsification conditions, the effects of the mass ratio of NP- 6 and $A B S C a, \mathrm{mR}_{\mathrm{OS}}$, stirring rate, the addition rate of water and the emulsification temperature on the mean droplet size of the nanoemulsion were investigated. Experimental results showed that the optimum emulsification conditions for the mass ratio of $\mathrm{NP}-6$ and $\mathrm{ABSCa}, \mathrm{mR}_{\mathrm{OS}}$, stirring rate, the addition rate of water and the emulsification temperature were respectively $5 / 5,1.4,8000 \mathrm{rpm}, 0.7 \mathrm{~mL} \min ^{-1}$ and $25{ }^{\circ} \mathrm{C}$. In addition, the bifenthrin nanoemulsion with the lower $\mathrm{mR}_{\mathrm{OS}}$ had a low interfacial tension and contact angle, which could be spread and wet on the leaf surface for the spraying application. 
The insecticidal activity of bifenthrin nanoemulsions against cabbage maggots was further studied, and the emulsion stability of the nanoemulsions against Ostwald ripening behavior was also evaluated. HPLC analysis showed that the bifenthrin nanoemulsion is not easily decomposed or precipitated, and has a good shelf life, making it suitable for potential pesticide formulations.

\section{Conflicts of interest}

There are no conflicts to declare.

\section{Acknowledgements}

We gratefully thank the financial support from the Natural Science Foundation of Hainan Province (218QN233), Scientific Research Projects of Hainan Higher Education Institutions (Hnky2019-36) and the National Natural Science Foundation of China (21566009).

\section{Notes and references}

1 M. S. El-Aasser and E. D. Sudol, JCT Res., 2004, 1, 21-31.

2 D. J. McClements and J. Rao, Crit. Rev. Food Sci. Nutr., 2011, 51, 285-330.

3 A. H. Saberi, Y. Fan and D. J. McClements, J. Agric. Food Chem., 2014, 62, 1625-1633.

4 M. Kah and T. Hofmann, Environ. Int., 2014, 63, 224-235.

5 Y. Liu, F. Wei, Y. Wang and G. Zhu, Colloids Surf., A, 2011, 389, 90-96.

6 Y. Chang and D. J. McClements, J. Agric. Food Chem., 2014, 62, 2306-2312.

7 O. Sonneville-Aubrun, J. T. Simonnet and F. Lalloret, Adv. Colloid Interface Sci., 2004, 108, 145-149.

8 L. Wang, X. Li, G. Zhang, J. Dong and J. Eastoe, J. Colloid Interface Sci., 2007, 314, 230-235.

9 S. Song, X. Liu, J. Jiang, Y. Qian, N. Zhang and Q. Wu, Colloids Surf., A, 2009, 350, 57-62.

10 N. Sadurní, C. Solans, N. Azemar and M. J. García-Celma, Eur. J. Pharm. Sci., 2005, 26, 438-445.

11 C. Bilbao-Sainz, R. J. Avena-Bustillos, D. Wood, T. G. Williams and T. H. Mchugh, J. Agric. Food Chem., 2010, 58, 11932-11938.

12 D. J. McClements, Soft Matter, 2012, 8, 1719-1729.

13 C. Solans and I. Solé, Curr. Opin. Colloid Interface Sci., 2012, 17, 246-254.

14 G. Calderó, M. J. García-Celma and C. Solans, J. Colloid Interface Sci., 2011, 353, 406-411.

15 F. Ostertag, J. Weiss and D. J. McClements, J. Colloid Interface Sci., 2012, 388, 95-102.

16 D. J. McClements, Soft Matter, 2011, 7, 2297-2316.

17 A. E. F. Abomohra, W. Jin, R. Tu, S. F. Han, M. Eid and H. Eladel, Renewable Sustainable Energy Rev., 2016, 64, 596606.

18 C. R. Soccol, C. J. D. Neto, V. T. Soccol, E. B. Sydney, E. S. F. da Costa, A. B. P. Medeiros and L. P. de S. Vandenberghe, Bioresour. Technol., 2017, 223, 259-268.
19 L. C. Jiang, M. Basri, D. Omar, M. B. A. Rahman, A. B. Salleh, R. N. Z. R. A. Rahman and A. Selamat, Pestic. Biochem. Physiol., 2012, 102, 19-29.

20 S. Song, X. Liu, J. Jiang, Y. Qian, N. Zhang and Q. Wu, Colloids Surf., A, 2009, 350, 57-62.

21 L. C. Peng, C. H. Liu, C. C. Kwan and K. F. Huang, Colloids Surf., A, 2010, 370, 136-142.

22 M. Jin, X. Zhang, L. Wang, C. Huang, Y. Zhang and M. Zhao, Aquat. Toxicol., 2009, 95, 347-354.

23 N. Ahmad, R. Ramsch, M. Llinàs, C. Solans, R. Hashim and H. A. Tajuddin, Colloids Surf., B, 2014, 115, 267-274.

24 W. Wang, H. Wei, Z. Du, X. Tai and G. Wang, ACS Sustainable Chem. Eng., 2015, 3, 443-450.

25 S. V. Joseph and J. Zarate, Crop Prot., 2015, 77, 148-156.

26 O. Sonneville-Aubrun, D. Babayan, D. Bordeaux, P. Lindner, G. Rata and B. Cabane, Phys. Chem. Chem. Phys., 2009, 11, 101-110.

27 A. H. E. Machado, D. Lundberg, A. J. Ribeiro, F. J. Veiga, B. Lindman, M. G. Miguel and U. Olsson, Langmuir, 2012, 28, 4131-4141.

28 C. J. Lim, M. Basri, D. Omar, M. B. A. Rahman, A. B. Salleh and R. N. Z. R. A. Rahman, Ind. Crops Prod., 2012, 36, 607613.

29 W. Warisnoicharoen, A. B. Lansley and M. J. Lawrence, Int. J. Pharm., 2000, 198, 7-27.

30 Y. He, B. Yang, G. Cheng and H. Pan, Mater. Lett., 2004, 58, 2019-2022.

31 K. Zielinska, K. A. Wilk, A. Jezierski and T. Jesionowski, J. Colloid Interface Sci., 2008, 321, 408-417.

32 P. Fernandez, V. Andre, J. Rieger and A. Kühnle, Colloids Surf., A, 2004, 251, 53-58.

33 Z. Hu, S. Ballinger, R. Pelton and E. D. Cranston, J. Colloid Interface Sci., 2015, 439, 139-148.

34 Y. Shen, Q. Zhao, X. Li, D. Yuan, Y. Hou and S. Liu, J. Hazard. Mater., 2012, 241-242, 472-477.

35 J. S. Yang, H. B. Ren and Y. J. Xie, Biomacromolecules, 2011, 12, 2982-2987.

36 K. Bouchemal, S. Briancon, E. Perrier and H. Fessi, Int. J. Pharm., 2004, 280, 241-251.

37 J. C. López-Montilla, P. E. Herrera-Morales, S. Pandey and D. O. Shah, J. Dispersion Sci. Technol., 2002, 23, 219-268.

38 Y. Chang, L. McLandsborough and D. J. McClements, J. Agric. Food Chem., 2013, 61, 8906-8913.

39 A. Shiloach and D. Blankschtein, Langmuir, 1998, 14, 71667182.

40 L. Yu, C. Li, J. Xu, J. Hao and D. Sun, Langmuir, 2012, 28, 14547-14552.

41 T. Tadros, P. Izquierdo, J. Esquena and C. Solans, Adv. Colloid Interface Sci., 2004, 108-109, 303-318.

42 I. Sole, C. M. Pey, A. Maestro, C. Gonzalez, M. Porras, C. Solans and J. M. Gutierrez, J. Colloid Interface Sci., 2010, 344, 417-423.

43 I. Capek, Adv. Colloid Interface Sci., 2004, 107, 125-155.

44 Z. Du, C. Wang, X. Tai, G. Wang and X. Liu, ACS Sustainable Chem. Eng., 2016, 4, 983-991. 